\title{
ANÁLISIS DEL DESEMPEÑO DE UN SISTEMA DE COMUNICACIÓN DE DA- TOS DE CORTO ALCANCE VÍA RADIO EN 2.4 GHZ BASADO EN ESPECTRO ENSANCHADO POR SECUENCIA DIRECTA (DSSS)
}

\section{PERFORMANCE ANALYSIS OF A SHORT RANGE 2.4 GHZ DIRECT SEQUENCE SPREAD SPECTRUM WIRELESS COMMUNICATION SYSTEM}

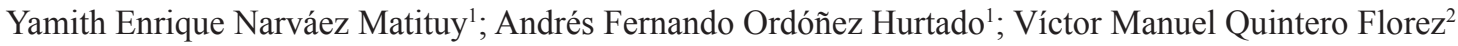

1 Grupo Radio e InALámbrias, GRIAL, Universidad del Cauca. yenaraez@unicauca.edu.co, afordonez@unicauca.edu.co.

2 Director del Grupo Radio e InALámbrias, GRIAL, Universidad del Cauca. vflorez@unicauca.edu.co.

\section{RESUMEN}

En el presente artículo se hace una introducción teórica acerca de la técnica de trasmisión de Espectro Ensanchado por Secuencia Directa (DSSS, Direct Sequence Spread Spectrum) y las ventajas obtenidas al modificar sus parámetros de configuración. Se presenta una descripción general del sistema de comunicación de datos vía radio de corto alcance en $2.4 \mathrm{GHz}$ basado en DSSS desarrollado a partir de los dispositivos de Radio Programables en un Chip (PRoC, Programmable Radio on Chip) de Cypress Semiconductor Corp. Una vez desarrollado el sistema de comunicación se diseñó un plan de pruebas para la evaluación y análisis de su desempeño a nivel físico con el fin de obtener medidas de potencia de recepción y número de errores de bits en la información recibida, a diferentes velocidades de transmisión de datos en tres escenarios específicos: exterior, interior y ante interferencia. Los resultados obtenidos permitieron realizar un análisis comparativo con el fin de determinar el comportamiento del sistema de comunicación ante diferentes condiciones de operación y en los diferentes escenarios.

Palabras Clave: DSSS, Sistema de Comunicación de Datos de Corto Alcance, PRoC, Desempeño, Nivel Físico, Interferencia.

\begin{abstract}
This paper contains a theoretical introduction about the Direct Sequence Spread Spectrum (DSSS) transmission technique, and the advantages obtained by modifying its configuration parameters. An overview about the short range $2.4 \mathrm{GHz}$ DSSS-based wireless communication system is presented, and its implementation in the Programmable Radio-on-Chip (PRoC) devices from Cypress Semiconductor Corp. A test plan was designed to evaluate and analyze the physical level performance of the system in order to obtain the received power measurements and number of bit errors in the received information, at different data transmission rates in three different scenarios: outdoor, indoor and interference-scenario. The obtained results allowed realize a comparative analysis to determine the behavior of the communication system under different operating conditions and in different scenarios.
\end{abstract}

Keywords: DSSS, Short range data communication system, PRoC, Performance, Physical level, Interference. 


\section{INTRODUCCIÓN}

La evolución de la electrónica y las telecomunicaciones ha permitido la creación de sistemas de comunicación robustos encargados de transmitir de manera confiable y efectiva grandes cantidades de información haciendo uso de técnicas de transmisión que mejoran considerablemente las características de operación de los sistemas. En este sentido, una de las áreas de las telecomunicaciones que ha tenido mayor crecimiento a nivel mundial ha sido el de las comunicaciones inalámbricas, lo que con el paso del tiempo ha impulsado la creación de redes que brindan movilidad, flexibilidad y escalabilidad. Sin embargo, los sistemas de comunicación inalámbrica presentan diferentes desventajas en cuanto a la disponibilidad del espectro radioeléctrico usado para la transmisión de las señales, la vulnerabilidad ante los efectos de ruido e interferencia en el entorno, el acceso al sistema por parte de usuarios no autorizados y la seguridad de la información transmitida, entre otras.

A medida que la tecnología ha avanzado se han desarrollado diferentes técnicas de transmisión de datos por radiofrecuencia que se encargan de mitigar los efectos mencionados anteriormente. Una de ellas es la técnica de transmisión DSSS, que consiste en la expansión en frecuencia de la señal de información a un valor mucho mayor que el requerido para transmitir la señal en banda base. Este proceso se denomina ensanchamiento y se logra a través de la combinación entre una señal de baja velocidad con una señal de alta velocidad denominada secuencia pseudo-aleatoria (1), conformada por una serie periódica de símbolos de corta duración denominados chips. En el receptor la señal recibida se combina de manera sincronizada con una réplica de la secuencia pseudo-aleatoria para recuperar la información original, siempre y cuando se conozca la secuencia correcta.

Debido las ventajas que ofrece esta técnica de transmisión, diferentes sistemas de comunicación hacen uso de ella con el propósito de incrementar su robustez y confidencialidad, entre los cuales se destacan los sistemas de comunicaciones móviles basados en la técnica de Acceso Múltiple por División de Código (CDMA, Code Division Multiple Access) (2).

En la Figura 1 se presenta la comparación entre el ancho de banda de la señal de información original y la señal de espectro ensanchado, cuya densidad espectral de potencia se encuentra por debajo del nivel del piso de ruido.

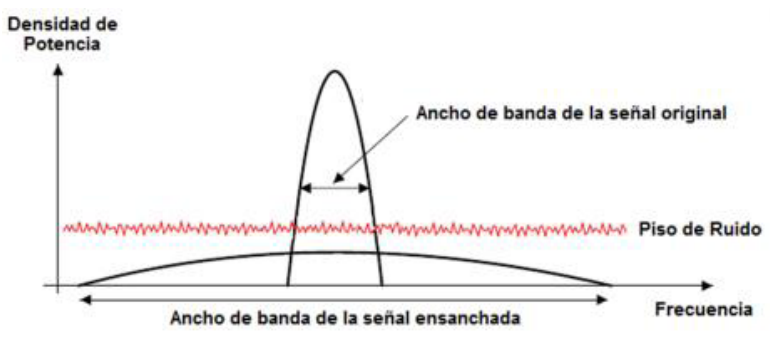

Figura 1. Ancho de Banda de la Señal Original y la de Espectro Ensanchado (3).

La relación que existe entre el ancho de banda de la información y el ancho de banda de la secuencia pseudoaleatoria se denomina Factor de Ensanchamiento (SF, Spreading Factor) o Ganancia de Procesamiento (PG, Processing Gain) y consiste en el número entero de chips a los que corresponde un bit de información (4), por lo que puede ser vista como la relación entre las velocidades de transmisión de información en chips con respecto a bits, como se muestra en [1].

$$
S F=\frac{B W_{c}}{B W_{b}}=\frac{R_{C}}{R_{b}}
$$

A mayor valor de SF se garantiza un mejor rechazo ante posible interferencia (4), debido a que cada elemento de la señal de información se representa por un número mayor de chips aparentemente aleatorios. Por esta razón se requieren características adecuadas de sincronismo en el receptor.

Para analizar el desempeño de un sistema de comunicación digital, en [2] se define la relación Energía de Bit $\left(E_{b}\right)$ con respecto a Densidad Espectral de Potencia de Ruido $\left(N_{0}\right)$ a la salida del receptor.

$$
\frac{E_{b}}{N_{0}}=\frac{P_{R} / B W_{b}}{P_{N} / B W_{c}}=\frac{P_{R}}{P_{N}} * \frac{B W_{c}}{B W_{b}}
$$

El cociente entre la potencia recibida y la potencia de ruido corresponde a la Relación Señal a Ruido (SNR, Signal-to-Noise Ratio) del canal de comunicación, mientras que la relación entre los anchos de banda corresponde al valor de SF. Por lo tanto, (3) se representa la relación $E_{b} / N_{0}$ en unidades logarítmicas.

$$
\left(\frac{E_{b}}{N_{0}}\right)_{d B}=(S N R)_{d B}+(S F)_{d B}
$$

Un receptor deseado puede aprovechar las ventajas de SF, permitiendo incrementar el valor de la potencia de la señal recibida por encima del nivel de ruido 
para recuperar la información, mientras que un receptor no deseado no cuenta con estas mismas capacidades. De acuerdo a esto se dice que los sistemas basados en DSSS cuentan con una baja probabilidad de interceptación de las señales, ya que se transmiten con muy baja densidad espectral de potencia, lo suficiente como para quedar enmascarada por el ruido de fondo del canal (5).

Adicionalmente, cuando una señal interferente de banda estrecha llega al receptor se combina con la secuencia pseudo-aleatoria, dando como resultado una señal de espectro ensanchado con un nivel de densidad espectral de potencia reducido, que será discriminada por un filtro pasa bajo encargado de remover las componentes no deseadas (3).

Gracias a estas y otras ventajas, la técnica de DSSS es el soporte principal de los sistemas CDMA, debido a que facilita la transmisión simultánea de señales de diferentes usuarios, compartiendo los mismos recursos de tiempo y frecuencia, diferenciándose unos de otros mediante la asignación de secuencias pseudo-aleatorias con valores pequeños de correlación cruzada u ortogonalidad entre sí (6).

Sin embargo, en los sistemas CDMA se presenta el fenómeno de Interferencia de Acceso Múltiple (MAI, Multiple Access Interference), que corresponde a la interferencia producida por los usuarios que transmiten simultáneamente su información dentro de un mismo ancho de banda (7). Este tipo de interferencia se genera si las secuencias pseudo-aleatorias no poseen las propiedades adecuadas de correlación cruzada u ortogonalidad.

\section{MATERIALES Y METODOS}

\section{Componentes del Sistema de Comunicación}

Los módulos de transmisión y recepción del sistema de comunicación se implementaron en cada uno de los dispositivos PRoC incluidos en el kit de desarrollo CY3653 a velocidades de transmisión de datos iguales a $16 \mathrm{kbps}, 32 \mathrm{kbps}$ y $64 \mathrm{kbps}$, correspondientes a valores de SF iguales a 64, 32 y 16, que generan una velocidad de transmisión de chips igual a 1 Mcps. Gracias a la programación adecuada de los microcontroladores fue posible aprovechar al máximo las ventajas de los transceptores radio basados en DSSS incluidos en los PRoC.

El módulo de transmisión es el encargado de realizar los procesos de ensanchamiento y modulación de la infor- mación, con el fin de lograr su transmisión de manera inalámbrica hasta el otro extremo, mientras que el de recepción se encarga de estimar la intensidad de la señal y detectar e interpretar la información recibida mediante los procesos inversos a los realizados en el transmisor.

El canal de transmisión corresponde al medio por el cual se propagan las señales de radiofrecuencia y en el cual ocurren variaciones en la potencia de la señal por efecto de las pérdidas de propagación que dependen de la distancia de separación entre el transmisor y el receptor, la frecuencia de operación y las características de los escenarios.

\section{Trabajo de Campo}

La evaluación y análisis del desempeño del sistema de comunicación implicó realizar mediciones de potencia de recepción y de cantidad de bits erróneos en la información recibida. Estas mediciones fueron realizadas en dos escenarios: un sector exterior despejado del Centro Deportivo Universitario (CDU) y el pasillo del tercer piso al interior del edificio de la Facultad de Ingeniería Electrónica y Telecomunicaciones (FIET) de la Universidad del Cauca, respectivamente.

Para analizar el desempeño a nivel físico del sistema de comunicación se diseñó un protocolo diferente al Wireless USB propio de los dispositivos $\mathrm{PRoC}$, de tal forma que se facilitó el envío y la recepción de la información. Para ello se requirió que la información transmitida viajara en forma de tramas que pudieran ser procesadas por el receptor para determinar la integridad de la información recibida, sin necesidad de retransmisiones ni confirmación de tramas recibidas.

\begin{tabular}{|c|c|c|c|c|c|}
\hline $\begin{array}{c}\text { Preámbulo } \\
24 \text { Bits }\end{array}$ & $\begin{array}{c}\text { Sincronización } \\
8 \text { Bits }\end{array}$ & $\begin{array}{c}\text { Longitud de } \\
\text { los datos } \\
8 \text { Bits }\end{array}$ & $\begin{array}{c}\text { Datos } \\
200 \text { Bits }\end{array}$ & $\begin{array}{c}\text { Checksum } \\
8 \text { Bits }\end{array}$ & $\begin{array}{c}\text { Campo } \\
\text { final } \\
8 \text { Bits }\end{array}$ \\
\hline
\end{tabular}

Figura 2. Tramas Implementadas en los Dispositivos PRoC.

El protocolo desarrollado se basa en la creación de tramas de información conformadas por seis campos de diferente tamaño, tal como se muestra en la Figura 2. La duración de cada uno de estos campos puede variar de acuerdo al número de chips que conforman cada bit de información, que corresponde al valor de SF, como se muestra en [4].

$$
\text { Duración de Trama }=256 \text { Bits } * T_{c} * S F
$$




\section{RESULTADOS Y DISCUSIÓN}

La evaluación del desempeño del sistema de comunicación implicó verificar las características de radiofrecuencia de los dispositivos $\mathrm{PRoC}$, realizar mediciones de potencia de recepción en los escenarios de interés, con el objetivo de determinar la relación $E_{b} / N_{0}$ y calcular el número de bits recibidos con errores. Todas las mediciones se realizaron en pasos de $1 \mathrm{~m}$ en cada escenario y fueron realizadas en repetidas ocasiones para obtener resultados válidos estadísticamente.

\section{A. Precisión y Estabilidad de la Frecuencia Por- tadora}

Dado que los dispositivos PRoC cuentan con un Oscilador de Cristal Compensado en Temperatura (TXCO, Temperature Crystal Compensated Oscillator), fue necesario analizar su comportamiento sometiendo el dispositivo a cambios de temperatura para determinar su precisión y estabilidad en la frecuencia de oscilación, que finalmente corresponde a la precisión de la portadora a nivel de radiofrecuencia, la cual se puede determinar mediante [5].

$$
\text { Precisión }[P P M]=\frac{\text { Corrimiento en Frecuencia }}{\text { Frecuencia teórica }} * 10^{6}
$$

Según el marcador M2 ubicado sobre el espectro de la Figura 3, la frecuencia portadora está localizada en $2.402024000 \mathrm{GHz}$. Es decir que presenta un corrimiento de $24 \mathrm{kHz}$ con respecto a la portadora teórica de $2.402 \mathrm{GHz}$. Por lo tanto, la precisión en la medida de la frecuencia portadora está dada por [6].

$$
\text { Precisión }[P P M]=\frac{24 \mathrm{kHz}}{2.402 \mathrm{GHz}} * 10^{6}=9.992
$$

De acuerdo con las especificaciones técnicas de los dispositivos PRoC, el oscilador presenta un margen de estabilidad en frecuencia de hasta \pm 30 PPM, que para aplicaciones de radiofrecuencia en 2.402 $\mathrm{GHz}$ corresponden a $\pm 72 \mathrm{kHz}$. A pesar de que existe un corrimiento de la frecuencia portadora en la situación anterior, este valor se encuentra dentro del margen permitido por el fabricante.

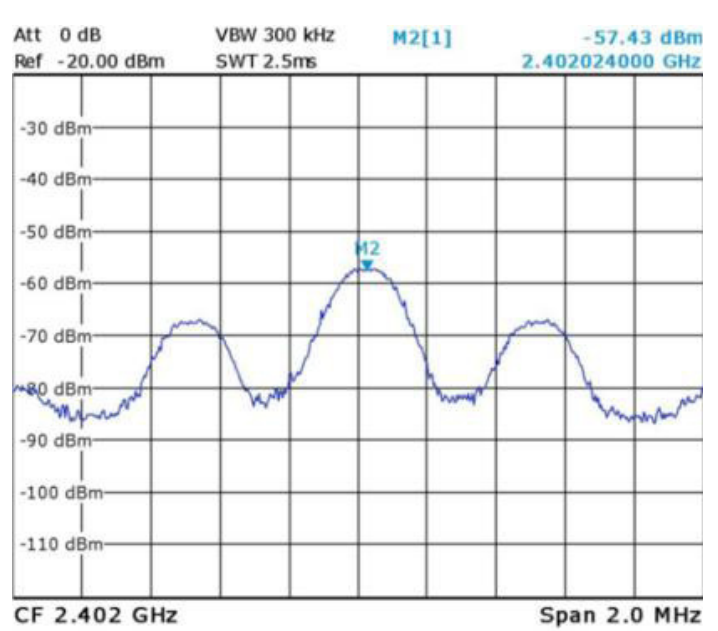

Figura 3. Espectro de la Frecuencia Portadora.

\section{B. Ancho de Banda de la Señal de Radiofrecuen- cia}

El ancho de banda útil de la señal transmitida se midió sobre el espectro de la señal de radiofrecuencia y corresponde a la separación entre los puntos en donde la densidad espectral de potencia se encuentra $6 \mathrm{~dB}$ por debajo del nivel de las componentes con mayor amplitud (8), que según la Figura 4 corresponden a la diferencia entre las frecuencias de los marcadores M1 y M2, dada por la expresión [7].

$$
B W[k H z]=M 2-M 1=958.11
$$

Este valor se aproxima al ancho de banda útil de $860 \mathrm{kHz}$ especificado por el fabricante (8), obteniendo un margen de error dado por [8].

$$
\text { Error }=\frac{|860 \mathrm{kHz}-958.11 \mathrm{kHz}|}{860 \mathrm{kHz}} * 100 \%=11.41 \text { [8] }
$$

\section{Potencia de Recepción}

A partir de los valores de sensibilidad y RSSI de referencia dados por el fabricante [8] se determinó una relación lineal entre los valores de RSSI con los valores de potencia en $\mathrm{dBm}$.

La comparación de los resultados de potencia de recepción obtenidos en los dos escenarios se muestra en la Figura 5, en donde es claro que en el es- 


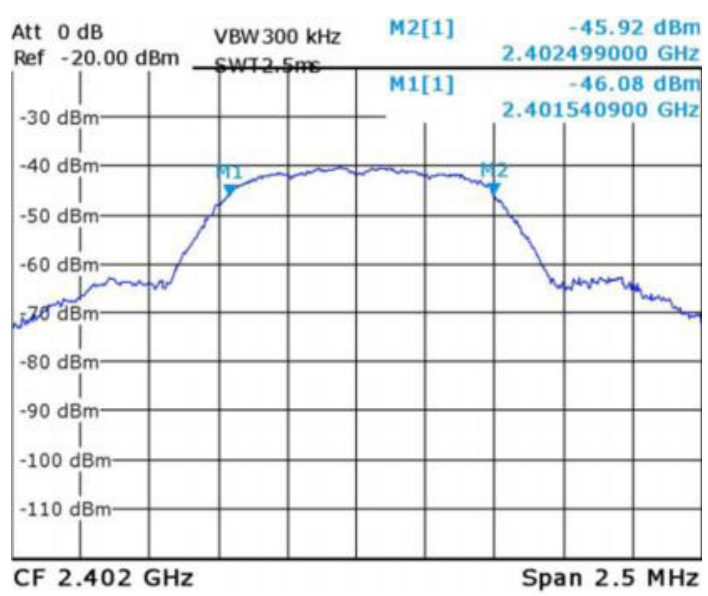

Figura 4. Ancho de Banda de Radiofrecuencia.

cenario interior se cubrieron distancias superiores que en el exterior. Este hecho se debe a que, a lo largo del pasillo la energía de la señal se encontraba confinada, lo cual pudo ser verificado matemáticamente haciendo uso del modelo de propagación de cañón o guía de onda (9), generado por la reflexión de las señales radioeléctricas en las paredes del pasillo.

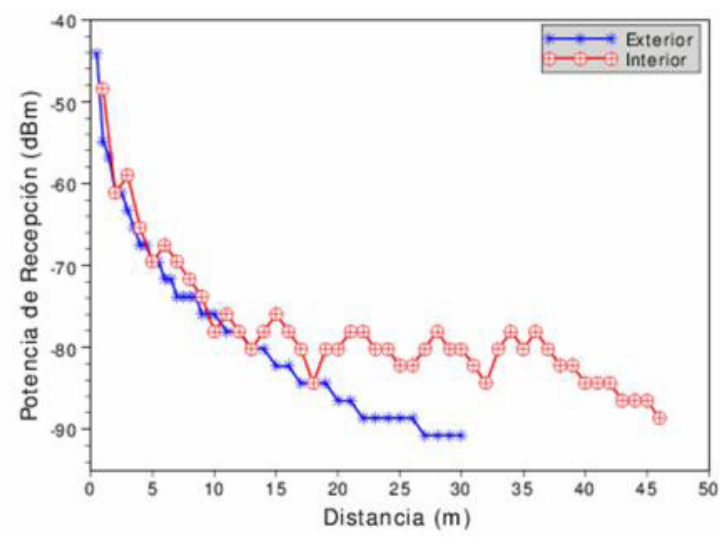

Figura 5. Potencia de Recepción en los Escenarios Exterior e Interior.

A pesar de que el sistema alcanzó una cobertura superior en el escenario interior, en algunos puntos de medición se presentaron desvanecimientos profundos, debido a que los ecos de una misma señal se interfierieron destructivamente por el efecto de multitrayectoria. A diferencia de este hecho, en el escenario exterior el transmisor radia en todas las direcciones, y tan solo una pequeña cantidad de la energía radiada es aprovechada por la antena del receptor.

\section{Evaluación y Análisis del Desempeño del Sis- tema de Comunicación}

Para realizar el análisis del desempeño del sistema de comunicación se dispone de la Tasa de Errores de Bit (BER, Bit Error Rate) que representa el número de bits erróneos con respecto al total de bits recibidos $^{2}$, como se muestra en [9].

$$
B E R=\frac{\text { Número de Bits Recibidos con Errores }}{\text { Númerode Bits Recibidos }}
$$

En los sistemas basados en DSSS es conveniente representar la calidad del canal de comunicación en términos de la relación energía de chips $\left(E_{c}\right)$ con respecto a la densidad de potencia de ruido denotada por $E_{c} / N_{o}$, que de acuerdo a [10] depende directamente del valor de SF.

$$
\left(\frac{E_{C}}{N_{0}}\right)_{d B}=\left(\frac{E_{b}}{N_{0}}\right)_{d B}-(S F)_{d B} \quad[10]
$$

\section{Análisis del Desempeño del Sistema en el Esce- nario Exterior}

Los resultados de BER en términos de la relación $E_{b} / N_{0}$ se muestran en la Figura 6, en la cual se observa un mejor desempeño del sistema al trabajar con una velocidad de transmisión de datos igual a

${ }^{2}$ Debido a que el sistema presenta la pérdida de un número considerable de tramas, se calcula la BER con respecto al total de bits recibidos 
$64 \mathrm{kbps}$, debido a que fue posible obtener una BER igual a $10^{-3}$ para un valor de $E_{b} / N_{0}$ cercano a 33 $\mathrm{dB}$, mientras que si el sistema operaba en las demás velocidades de transmisión alcanzar el valor de $10^{-3}$ requería valores superiores de $E_{b} / N_{0}$.

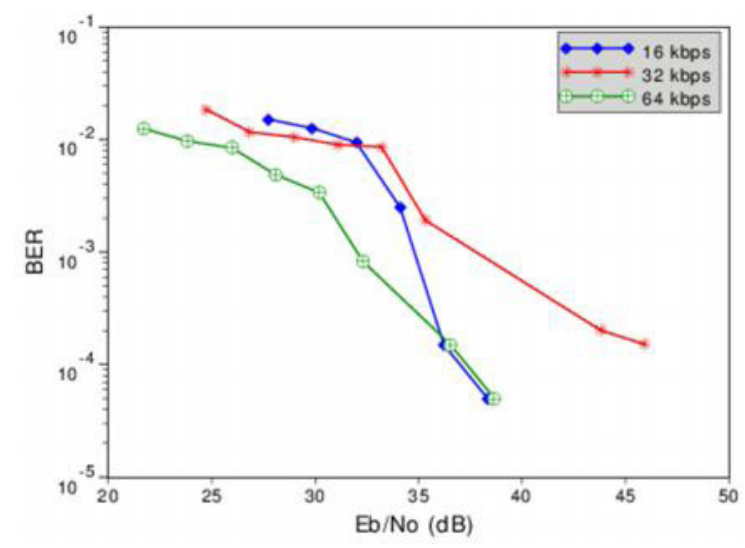

Figura 6. BER vs $E_{b} / N_{0}$ en el Escenario Exterior.

En la Figura 7 se muestra la BER del sistema en el escenario exterior en función de la relación $E_{c} /$ $N_{0}$ para cada una de las velocidades de transmisión de datos del sistema, en donde se observa un mejor desempeño del sistema a una velocidad de transmisión de datos igual a 16 kbps.

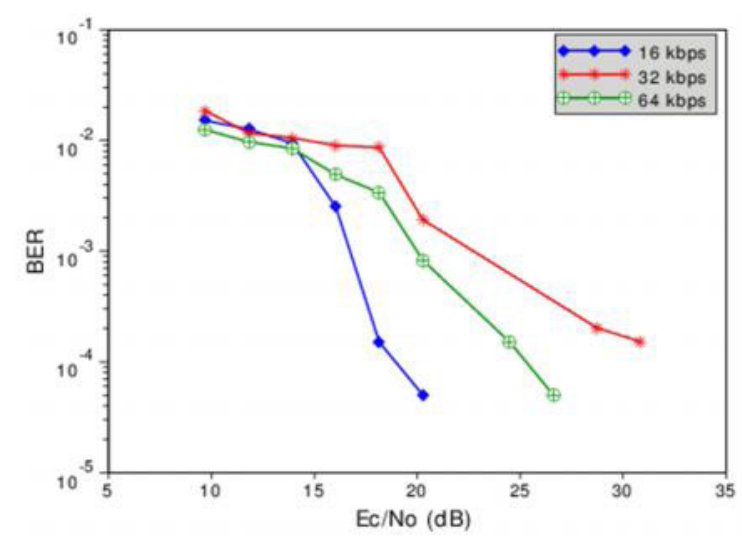

Figura 7. BER vs $E_{c} / N_{0}$ en el Escenario Exterior.

Es claro que los valores de $E_{c} / N_{0}$ se encuentran por debajo de los valores de $E_{b} / N_{0}$, ya que de acuerdo a [10] la ganancia adicional del sistema dada por SF origina un desplazamiento negativo de las curvas en el eje horizontal. Por esta razón se observa que la curva correspondiente a $16 \mathrm{kbps}$ presenta un mayor corrimiento, dando mejores resultados a nivel de chip.

\section{Análisis del Desempeño del Sistema en el Esce- nario Interior}

Los resultados en términos de $E_{b} / N_{0}$ para este escenario se muestran en las Figura 8, donde se observa que al igual que en el escenario exterior se obtuvo un mejor desempeño para la velocidad de transmisión de datos igual a $64 \mathrm{kbps}$, ya que se lograron resultados de BER de $10^{-3}$ para valores de $E_{b} / N_{0}$ cercanos a $35 \mathrm{~dB}$, mientras que si el sistema operaba en las demás velocidades se requerían valores superiores de $E_{b} / N_{0}$ para conseguir el mismo valor de BER.

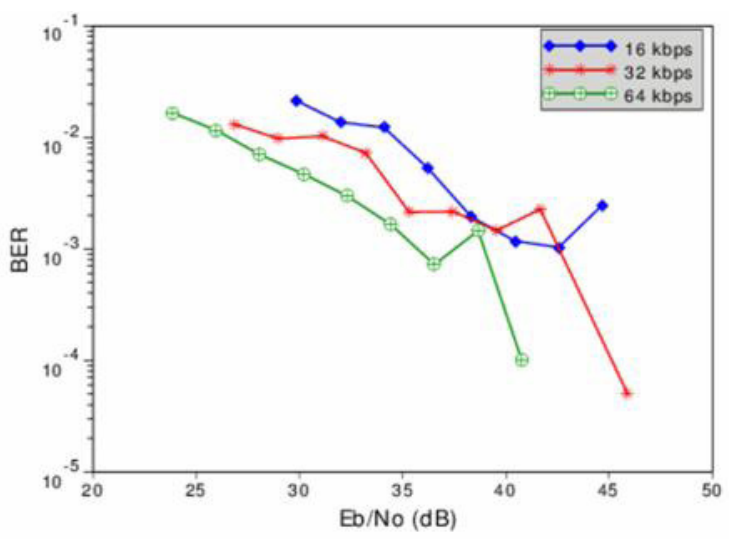

Figura 8. BER vs $E_{b} / N_{0}$ en el Escenario Interior.

En algunos casos se obtuvo un desempeño menos favorable para la velocidad de transmisión de 16 kbps, ya que este escenario está afectado en gran medida por la interferencia causada por el multitrayecto, que según la Figura 8 incrementa la BER en algunos puntos del escenario.

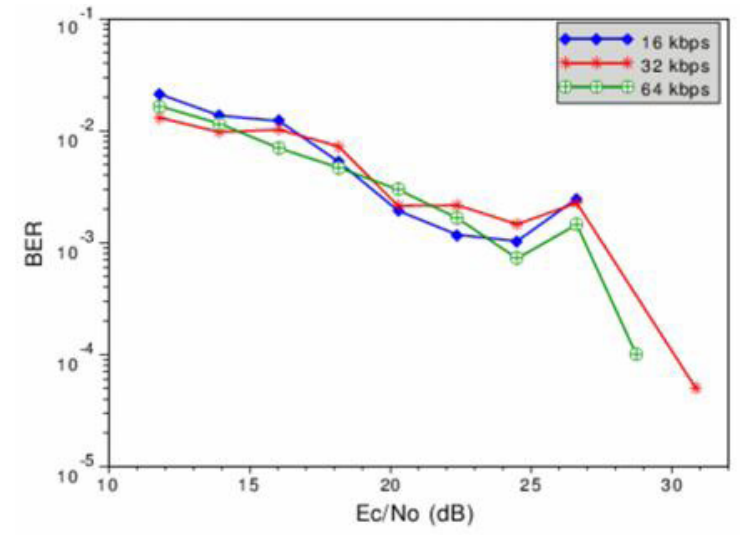

Figura 9. BER vs $E_{c} / N_{0}$ en el Escenario Interior. 
En la Figura 9 se muestra la BER del sistema en el escenario interior en función de la relación $E_{c} / N_{0}$ para cada una de las velocidades de transmisión de datos del sistema, donde se observa que el comportamiento del sistema es relativamente similar para las tres velocidades de transmisión de datos, para valores de $E_{c} / N_{0}$ menores a $25 \mathrm{~dB}$.

En algunos puntos del pasillo ocurre un incremento del nivel de potencia de las señales recibidas debido a las condiciones de multitrayecto a lo largo del pasillo, lo cual permite aumentar el nivel de $E_{c} / N_{0}$ y de la misma manera reducir la BER del sistema.

\section{Análisis del Desempeño del Sistema en el Esce- nario ante Interferencia}

El parámetro utilizado para evaluar el desempeño de un sistema DSSS en un ambiente de interferencia corresponde a la relación $E_{c}$ con respecto a interferencia $\left(I_{0}\right)$, definido en [11].

$$
\frac{E_{c}}{I_{0}}=\frac{P_{R} / B W_{c}}{P_{I} / B}=\frac{P_{R}}{P_{I}}=S I R
$$

Con el objetivo de generar niveles de interferencia en la misma frecuencia de operación del sistema de comunicación, se dispuso de un enrutador inalámbrico transmitiendo en el canal 11 de Wi-Fi, correspondiente a $2.462 \mathrm{GHz}$, y uno de los dispositivos Nordic nRF24LU1-DK operando a una frecuencia igual $2.478 \mathrm{GHz}$.

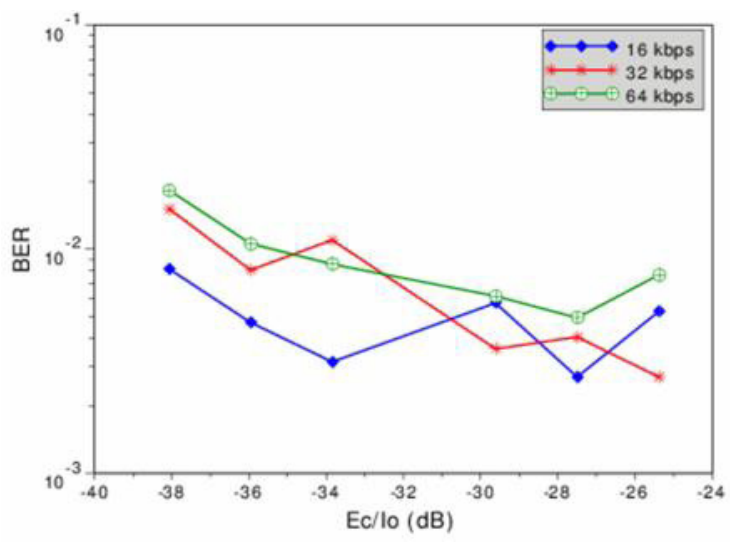

Figura 10. BER vs $E_{c} / I_{0}$ en el Escenario ante Interferencia del Enrutador Inalámbrico.
De la Figura 10 se observa que el sistema presentó un mejor desempeño en $16 \mathrm{kbps}$ en el escenario ante la interferencia del enrutador, ya que existe mayor probabilidad de recuperar la información original si se dispone de un número superior de chips por cada bit, lo que mejora las capacidades para realizar la correlación de las señales de espectro ensanchado en el receptor, incluso cuando se cuenta con niveles elevados de interferencia.

A diferencia de la situación anterior, en la Figura 11 se observa que el sistema aparentemente presentó un comportamiento menos favorable para la velocidad de transmisión de datos de $16 \mathrm{kbps}$, mientras que para la velocidad de $64 \mathrm{kbps}$ se obtuvieron mejores resultados de desempeño, ya que la BER se mantiene alrededor de $10^{-3}$.

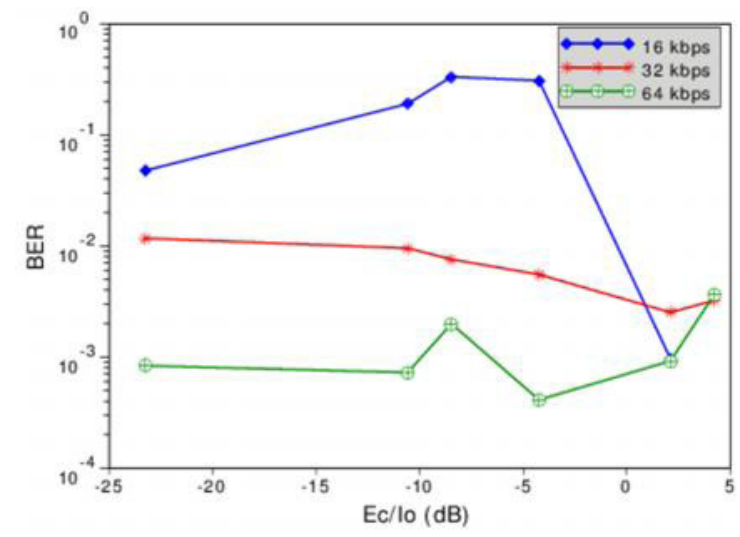

Figura 11. BER vs $E_{c} / I_{0}$ en el Escenario ante Interferencia del Dispositivo Nordic.

Las señales transmitidas por los dispositivos Nordic interfirieron en mayor proporción al sistema de comunicación, ya que el ancho de banda en donde se concentraba la energía de las señales interferentes fue comparable con el ancho de banda de las señales deseadas, lo cual redujo notoriamente el desempeño del sistema.

En ambas situaciones de interferencia se obtuvieron valores negativos de $E_{c} / I_{0}$, lo cual indica que la potencia de la interferencia medida en el receptor fue mayor que la potencia de las señales deseadas, lo que ocasionó el enmascaramiento de las señales de interés por parte de las señales interferentes. Debido a esto es necesario incrementar la potencia de transmisión para que el sistema tenga un mejor 
comportamiento ante la interferencia causada por otros sistemas.

\section{CONCLUSIONES}

La implementación de un sistema de comunicación de datos de corto alcance vía radio en $2.4 \mathrm{GHz}$ basado en DSSS mediante dispositivos PRoC requiere de la configuración adecuada de sus parámetros para satisfacer las exigencias de calidad y cobertura.

De acuerdo al análisis del sistema de comunicación, se pudo determinar que su desempeño a nivel físico fue aceptable, ya que los resultados obtenidos indican que se lograron menores valores de BER para una velocidad de transmisión de 64 kbps. Por lo tanto es posible realizar la implementación de diferentes sistemas funcionando a la máxima velocidad de transmisión permitida para los dispositivos PRoC.

Teóricamente el sistema implementado es capaz de soportar más errores a una velocidad de transmisión de $16 \mathrm{kbps}$, pues se cuenta con un número mayor de chips por cada bit. En las medidas obtenidas en el escenario ante interferencia del enrutador inalámbrico se obtuvo este comportamiento, lo cual indica que los dispositivos PRoC son capaces de soportar la interferencia producida por elementos que operan bajo el estándar Wi-Fi.

\section{AGRADECIMIENTOS}

Los autores agradecen a la Universidad del Cauca, la Vicerrectoría de Investigaciones, la Facultad de Ingeniería Electrónica y Telecomunicaciones, el Departamento de Telecomunicaciones y sus grupos de investigación Grupo de Radio e InALámbricas (GRIAL) y Grupo I+D Nuevas Tecnologías en Telecomunicaciones (GNTT) por su apoyo.

Este artículo es el resultado de un trabajo de grado, el cual a su vez hizo parte del proyecto de investigación “PROTOTIPO DE COMUNICACIÓN DE DATOS DE CORTO ALCANCE EN 2.4 GHZ” con código 3085, financiado por la Vicerrectoría de Investigaciones de la Universidad del Cauca

\section{BIBLIOGRAFIA}

1. Ziemer, R. Fundamentals of Spread Spectrum Modulation. United States of America: Morgan \& Claypool Publishers, 2007; pp 1-10.

2. Dinan, E. and Jabbari, B. Spreading Codes for Direct Sequence CDMA and Wideband CDMA Cellular Networks, IEEE Communication Magazine, 1998; 36, pp. 48-54.

3. Fazel, K. and Kaiser, S. Multi-Carrier and Spread Spectrum Systems. England: John Wiley \& Sons, Ltd., 2003; pp. 32-35.

4. Torrieri, D. Principles of Spread Spectrum Modulation Systems. United States of America: Springer Science + Business Media Inc., 2005; pp. 55-60.

5. Proakis, J. and Saheli, M. Communication Systems Engineering. United States of America: Prentice Hall Inc., 1994; pp. 742-748.

6. Cooper, G. and McGillem, C. Modern Communications and Spread Spectrum. United States of America: McGraw-Hill Inc., 1986.

7. Holma, H. and Toskala, A. WCDMA for UMTS: Radio Access for Third Generation Mobile Communications. England: Wiley \& Sons, Ltd., 2004; pp. 398-401.

8. Cypress Semiconductor, "CYWUSB6935 WirelessUSB LR 2.4-GHz DSSS Radio SoC", Cypress Semiconductor Corp. United States of America: 2004.

9. Goldsmith, A. Wireless Communications. United States of America: Cambridge University Press, 2005; pp. 33-36. 